\title{
The onset of the magnetic buoyancy instability in partially ionized plasmas
}

\begin{abstract}
G. T. Birk ${ }^{\star}$
Institute for Astronomy and Astrophysics, University of Munich, Germany

Centre of Interdisciplinary Plasma Science, Garching, Germany

Received 16 April 2002 / Accepted 4 June 2002

Abstract. The magnetic buoyancy instability greatly influences the transport of magnetic flux in a variety of astrophysical objects. In this paper the stability of magnetic flux layers against magnetic buoyancy perturbations is studied for the first time for partially ionized plasmas. Different to the pure magnetohydrodynamic case, the onset of unstable modes depends not only on the field gradient but also on the field strength. Momentum transfer between the ionized and neutral plasma components leads to stabilization of at least short wavelengths or even all buoyancy modes.
\end{abstract}

Key words. instabilities - sunspots - galaxies: intergalactic medium- galaxies: magnetic fields

\section{Introduction}

Macroscopic instabilities due to magnetic buoyancy are very important in astrophysical plasmas, in particular, in the context of the formation of sun spots and the transport of magnetic flux from galactic disks and accretion disks in the halos and coronae (e.g. Priest 1982; Tajima \& Shibata 1997 and references therein). Consequently, since the pioneering work by Schatzman (1963) and Parker (1966), exhaustive analytical (e.g. Hanasz \& Lesch 1993; Giz \& Shu 1993; Foglizzo \& Tagger 1994; Kamaya et al. 1997; Kim \& Ryu 2001) and numerical (e.g. Matsumoto et al. 1990; Matsuzaki et al. 1997; Santillán et al. 2000; Kim et al. 2001) studies in the framework of magnetohydrodynamics (MHD) have result in a detailed understanding of many aspects of the linear and nonlinear dynamics of the unstable rise of magnetic flux systems in gravitational fields. However, the considered instability, usually named Parker instability, very often operates in partially ionized plasmas rather than in fully ionized ones and, strictly speaking, only for the latter does a pure MHD treatment seem appropriate. In molecular clouds and accretion disks a neutral gas component takes part in the macroscopic dynamics. Up to now no investigations of the Parker instability in a partially ionized two-fluid plasma can be found in the literature. In this work the onset of unstable modes due to magnetic buoyancy is studied by a self-consistent set of balance equations for the neutral gas and ionized components and the magnetic field. In the following section the macroscopic multi-fluid description is introduced. In Sect. 3 the onset criterion for the magnetic buoyancy instability in partially ionized plasmas is derived by means of a linear mode analysis.

\footnotetext{
* e-mail: birk@usm.uni-muenchen.de
}

\section{The two-fluid model}

The macroscopic isothermal low-frequency dynamics of a partially ionized plasma in ionization equilibrium is governed by the following balance equations. The mass density balance equations read

$\frac{\partial \rho}{\partial t}=-\nabla \cdot(\boldsymbol{v} \rho)$

and

$\frac{\partial \rho_{n}}{\partial t}=-\nabla \cdot\left(\boldsymbol{v}_{n} \rho_{n}\right)$

where $\rho$ and $v$ denote the mass densities and bulk velocities. Here and in the following equations the index $n$ denotes the neutral gas component. Momentum transport is governed by

$\frac{\partial}{\partial t}(\rho \boldsymbol{v})=-\nabla \cdot(\rho \boldsymbol{v} \boldsymbol{v})-\nabla p+\frac{1}{\mu}(\nabla \times \boldsymbol{B}) \times \boldsymbol{B}-\rho v\left(\boldsymbol{v}-\boldsymbol{v}_{n}\right)$

and

$\frac{\partial}{\partial t}\left(\rho_{n} \boldsymbol{v}_{n}\right)=-\nabla \cdot\left(\rho_{n} \boldsymbol{v}_{n} \boldsymbol{v}_{n}\right)-\nabla p_{n}+\rho v\left(\boldsymbol{v}-\boldsymbol{v}_{n}\right)$

where $p, \boldsymbol{B}$, and $v$ are the thermal pressure, magnetic field and effective plasma-neutral gas collision frequency. The temporal evolution of the magnetic field is given by the induction equation

$\frac{\partial \boldsymbol{B}}{\partial t}=\nabla \times(\boldsymbol{v} \times \boldsymbol{B})$

where the pressure term, the Hall term, the resistive term and the magnetic field self-generation term due to relative shear flows are neglected for simplicity. The system of equations can be closed by adiabatic equations of state or, as in our case, by the ideal gas equations

$p=\frac{\rho}{m} T ; p_{n}=\frac{\rho_{n}}{m_{n}} T_{n}$ 
where $T$ (measured in energy units) and $m$ are the temperature and particle mass. In order to perform a normal mode analysis these equations have to be linearized. The initial stationary state has to satisfy the corresponding time-independent balance equations. Here, we concentrate on a static isothermal plasma-neutral gas system characterized by $\rho_{0}(z), p_{0}(z)$, $T_{0}(z)$ and $\rho_{n 0}(z), p_{n 0}, T_{n 0}(z)$ as well as $\boldsymbol{B}_{0}=B_{0} \boldsymbol{e}_{x}$. In the present study, we do not have to specify the actual equilibrium profiles. For the perturbations of the equilibrium we choose the standard normal mode ansatz of localized Fourier modes $\Psi=\hat{\Psi} \exp \left(\omega t+i k_{x} x+i k_{y} y\right)$. Our stability analysis follows closely the MHD one performed by Gilman (1970).

\section{The linearization and the onset criterion}

Linearization of Eqs. (1)-(5) and use of the normal modes ansatz results in

$\omega \hat{\rho}=-i \rho_{0}\left(k_{x} \hat{v}_{x}+k_{y} \hat{v}_{y}\right)-\frac{\partial}{\partial z}\left(\rho_{0} \hat{v}_{z}\right)$

$\omega \hat{\rho}_{n}=-i \rho_{n 0}\left(k_{x} \hat{v}_{n x}+k_{y} \hat{v}_{n y}\right)-\frac{\partial}{\partial z}\left(\rho_{n 0} \hat{v}_{n z}\right)$

$\omega \rho_{0} \hat{v}_{x}=-i k_{x} \hat{\rho} \frac{T_{0}}{m}+\frac{\hat{B}_{z}}{\mu} \frac{\partial B_{0}}{\partial z}-v \rho_{0}\left(\hat{v}_{x}-\hat{v}_{n x}\right)$

$\omega \rho_{0} \hat{v}_{y}=i k_{x} \hat{B}_{y} \frac{B_{0}}{\mu}-i k_{y}\left(\hat{\rho} \frac{T_{0}}{m}+\hat{B}_{x} \frac{B_{0}}{\mu}\right)-v \rho_{0}\left(\hat{v}_{y}-\hat{v}_{n y}\right)$

$\omega \rho_{0} \hat{v}_{z}=i k_{x} \hat{B}_{z} \frac{B_{0}}{\mu}-\frac{\partial}{\partial z}\left(\hat{\rho} \frac{T_{0}}{m}+\hat{B}_{x} \frac{B_{0}}{\mu}\right)-\hat{\rho} g-v \rho_{0}\left(\hat{v}_{z}-\hat{v}_{n z}\right)$

$\omega \rho_{n 0} \hat{v}_{n x}=-i k_{x} \hat{\rho}_{n} \frac{T_{n 0}}{m_{n}}+v \rho_{0}\left(\hat{v}_{x}-\hat{v}_{n x}\right)$

$\omega \rho_{n 0} \hat{v}_{n y}=-i k_{y} \hat{\rho}_{n} \frac{T_{n 0}}{m_{n}}+v \rho_{0}\left(\hat{v}_{y}-\hat{v}_{n y}\right)$

$\omega \rho_{n 0} \hat{v}_{n z}=-\frac{\partial}{\partial z} \frac{\hat{\rho}_{n} T_{n 0}}{m_{n}}-\hat{\rho}_{n} g+v \rho_{0}\left(\hat{v}_{z}-\hat{v}_{n z}\right)$

$\omega \hat{B}_{x}=-i B_{0} k_{y} \hat{v}_{y}-\frac{\partial}{\partial z}\left(B_{0} \hat{v}_{z}\right)$

$\omega \hat{B}_{y}=-i B_{0} k_{x} \hat{v}_{y}$

$\omega \hat{B}_{z}=-i B_{0} k_{x} \hat{v}_{z}$

where the perturbed pressures have been replaced by the perturbed mass densities according to Eq. (6). Equations (7) and (15) can be combined to give

$\omega \hat{B}_{x}-i k_{x} B_{0} \hat{v}_{x}-\omega \frac{B_{0}}{\rho_{0}} \hat{\rho}-\left(B_{0} \frac{\mathrm{d}}{\mathrm{d} z} \ln \rho_{0}-\frac{\partial B_{0}}{\partial z}\right) \hat{v}_{z}=0$.
If one concentrates on the most unstable modes (see Parker \& Jokipii 2000) that are very localized in the $y$-direction, i.e. formally $k_{y} \rightarrow \infty$, Eq. (10) demands, for the lateral pressure balance in the ionized fluid component,

$\hat{\rho} \frac{T_{0}}{m}+\hat{B}_{x} \frac{B_{0}}{\mu}=0$.

Consequently, the respective term in Eq. (11) must also vanish. From Eq. (13) one finds that $\hat{\rho}_{n}=0$ in order to avoid an infinite oscillation frequency of the perturbations. Equations (12) and (14) can be used to eliminate $\hat{v}_{n x}$ and $\hat{v}_{n z}$ from Eqs. (9) and (11)

$$
\begin{aligned}
i k_{x}\left(\omega \rho_{n 0}+v \rho_{0}\right) c_{\mathrm{s}}^{2} \hat{\rho}+ & \omega \rho_{0}\left((\omega+v) \rho_{n 0}+v \rho_{0}\right)= \\
& \hat{v}_{x}-\left(\omega \rho_{n 0}+v \rho_{0}\right) \frac{1}{\mu} \frac{\partial B_{0}}{\partial z} \hat{B}_{z}=0
\end{aligned}
$$

$g\left(\omega \rho_{n 0}+v \rho_{0}\right) \hat{\rho}+\omega \rho_{0}\left((\omega+v) \rho_{n 0}+v \rho_{0}\right) \hat{v}_{z}$

$$
-i k_{x} B_{0}\left(\omega \rho_{n 0}+v \rho_{0}\right) \frac{1}{\mu} \hat{B}_{z}=0
$$

with the local isothermal sound speed $c_{\mathrm{S}}=\sqrt{T_{0} / m}$. Thus, the present stability analysis reduces to the solutions of the decoupled homogeneous algebraic system of the five Eqs. (17)-(21) for the unknown perturbation amplitudes $\hat{\rho}, \hat{v}_{x}, \hat{v}_{z}, \hat{B}_{x}$ and $\hat{B}_{z}$. Non-trivial solutions demand for the vanishing of the coefficient determinant

\section{See Eq. (22) above.}

The characteristic polynomial is of the 6th order in $\omega$ and reduces to the well-known 4th order polynomial and the classical onset criterion of instability (Gilman 1970) for vanishing plasma-neutral gas interactions. Since the characteristic polynomial from Eq. (22) has only real coefficients, the classical Hurwitz (1895) method can be used to find the conditions for positive roots, i.e. instability (see also, e.g., Giaretta 1979). The second Hurwitz sub-determinant provides us with the criterion for the instability of magnetic buoyancy modes in partially ionized plasmas

$$
\begin{aligned}
0 & >\rho_{n 0}^{3}\left(\frac{v_{\mathrm{A}}^{2}}{c_{\mathrm{s}}^{2}}+1\right) \frac{g}{c_{\mathrm{s}}^{2}} \frac{\mathrm{d}}{\mathrm{d} z} \ln B_{0}+\rho_{n 0}^{3}\left(\frac{v_{\mathrm{A}}^{4}}{c_{\mathrm{s}}^{4}}+3 \frac{v_{\mathrm{A}}^{2}}{c_{\mathrm{s}}^{2}}+2\right) k_{x}^{2} \\
& +\left[\left(6\left(\rho_{0}^{2} \rho_{n 0}+\rho_{0} \rho_{n 0}^{2}\right)+2\left(\rho_{0}^{3}+\rho_{n 0}^{3}\right)\right)\left(\frac{v_{\mathrm{A}}^{2}}{c_{\mathrm{s}}^{4}}+\frac{2}{c_{\mathrm{s}}^{2}}\right)\right. \\
& \left.+\left(2\left(\rho_{0}^{3}+\rho_{n 0}^{3}\right)+6\left(\rho_{0} \rho_{n 0}^{2}+\rho_{0}^{2} \rho_{n 0}\right)\right) \frac{1}{v_{\mathrm{A}}^{2}}\right] v^{2} \\
& -\left(\frac{v_{\mathrm{A}}^{2}}{c_{\mathrm{s}}^{4}}+\frac{1}{c_{\mathrm{s}}^{2}}\right) g \rho_{n 0}^{3} \frac{\mathrm{d}}{\mathrm{d} z} \ln \rho_{0} .
\end{aligned}
$$

Note that the destabilizing effect of an upward-directed positive mass density gradient is associated with convection which 
is a process we are not interested in here. The buoyancy instability is driven by the first term of Eq. (23) for a magnetic field that decreases with height, but the criterion for the wave numbers of unstable modes is much more complicated than in the pure MHD case. First, one identifies the stabilizing effect of the collisional plasma-neutral gas momentum transfer due to the $v^{2}$-term in Eq. (23). Moreover, the onset criterion depends on the strength of the magnetic field and not only on the field gradient. In the low- $\beta$ limit $\left(c_{\mathrm{s}}^{2} \gg v_{\mathrm{A}}^{2}\right)$ the onset criterion reads

$$
\begin{aligned}
k_{x}^{2}+\left(\left(\rho_{0}^{3}+\rho_{n 0}^{3}\right)+3\left(\rho_{0} \rho_{n 0}^{2}+\rho_{0}^{2} \rho_{n 0}\right) \frac{v^{2}}{v_{\mathrm{A}}^{2} \rho_{n 0}^{3}}\right. & -\frac{g}{2 c_{\mathrm{s}}^{2}} \frac{\mathrm{d}}{\mathrm{d} z} \ln \rho_{0} \\
& <-\frac{g}{2 c_{\mathrm{s}}^{2}} \frac{\mathrm{d}}{\mathrm{d} z} \ln B_{0} .
\end{aligned}
$$

On the other hand, in the high- $\beta$ limit $\left(c_{\mathrm{s}}^{2} \ll v_{\mathrm{A}}^{2}\right)$ unstable mode are excited, if

$$
\begin{aligned}
k_{x}^{2}+\left(6\left(\rho_{0}^{2} \rho_{n 0}+\rho_{0} \rho_{n 0}^{2}\right)+2\left(\rho_{0}^{3}+\rho_{n 0}^{3}\right)\right) \frac{v^{2}}{v_{\mathrm{A}}^{2} \rho_{n 0}^{3}} \\
-\frac{g}{v_{\mathrm{A}}^{2}} \frac{\mathrm{d}}{\mathrm{d} z} \ln \rho_{0}<-\frac{g}{v_{\mathrm{A}}^{2}} \frac{\mathrm{d}}{\mathrm{d} z} \ln B_{0} .
\end{aligned}
$$

\section{Conclusions}

Emerging flux tubes in molecular clouds, accretion and galactic disks as well as in the solar lower photosphere can be interpreted as the result of magnetic buoyancy instabilities. In these partially ionized astrophysical plasmas the effect of a neutral gas component is of importance for the macroscopic dynamics but is usually neglected for simplicity. In the present study it is shown that the onset criterion for the Parker instability is significantly modified in partially ionized systems as compared to pure magnetohydrodynamic ones. In particular, the onset of unstable buoyancy modes does not only depend on the magnetic field gradient but also the on the field strength. This holds even in low- $\beta$ plasmas. Short wavelengths are stabilized due to collisional momentum transfer.
Depending on the actual plasma parameters the buoyancy instability may even be completely suppressed.

In the present analysis a rather simple version of Ohm's law is used. It should be of interest to include generalizations like the Hall effect and pressure gradients in the induction equation.

The multi-fluid treatment shows that one has to act with caution in interpreting emerging magnetic flux phenomena in partially ionized astrophysical systems in terms of the Parker instability. At least as far as the linear stability is concerned, one has to check carefully whether or not the onset criterion can be fulfilled for the observed spatial scales. Non-linear studies are a promising task for the future.

\section{References}

Foglizzo, T., \& Tagger, M. 1994, A\&A, 301, 293

Giaretta, D. L. 1979, A\&A, 75, 273

Gilman, P. A. 1970, ApJ, 162, 1019

Giz, A. T., \& Shu, F. H. 1993, ApJ, 404, 185

Hanasz, M., \& Lesch, H. 1997, A\&A, 321, 1007

Hurwitz, A. 1895, Math. Ann., 46, 273

Kamaya, H., Horiuchi, T., Matsumoto, R., et al. 1997, ApJ, 485, 228

Kim, J., Ryu, D., \& Jones, T. W. 2001, ApJ, 557, 464

Kim, J., \& Ryu, D. 2001, ApJ, 561, L135

Matsumoto, R., Hanawa, T. Shibata, K., \& Horiuchi, T. 1990, ApJ, 356,259

Matsuzaki, T., Matsumoto, R., Tajima, T., \& Shibata, K. 1997, in Accretion Phenomena and Related Outflows, IAU Colloq. 163, ASP Conf. Ser. 121, ed. D. T. Wickramasinghe, G. V. Bicknell, \& L. Ferrario, 766

Parker, E. N. 1966, ApJ, 145, 811

Parker, E. N., \& Jokipii, J.R. 2000, ApJ, 536, 331

Priest, E. R. 1982, Solar Magnetohydrodynamics (Reidel, Dordrecht), Ch. 8

Santillán, A., Kim, J., Franco, J., et al. 2000, ApJ, 545, 353

Schatzman, E. 1963, Ann. Astrophys., 26, 166

Tajima, T., \& Shibata, K. 1997, Plasma Astrophysics (AddisonWesley, Reading), Ch. 3 